\section{Measuring Adherence to Antihypertensive Drug Therapy}

To the Editor:

Adherence to therapy as measured in a controlled clinical environment may not reflect adherence in usual-care settings because of the multifactorial nature of adherence. Thus, for large population studies, where the more expensive and timeconsuming methods like pill counts, assessment of drug levels, and patient self-report are not feasible, refill compliance (compliance as measured from pharmacy refill records) may provide otherwise unattainable information about the true nature of adherence to therapy. ${ }^{1}$ In the article on patient adherence with amlodipine, lisinopril, or valsartan therapy in a usual-care setting, the authors have assessed adherence and persistence from a pharmacy claims database utilizing a retrospective cohort study design with a 2-year follow-up. ${ }^{2}$ Although this approach is appropriate and inexpensive for a large-population-based study, we would like to draw attention to certain points that may be relevant when interpreting the study results.

Data for the study were obtained from a large pharmacy benefits manager with a diverse payer mix. Although the authors address this issue as an advantage, probably pertaining to the external validity of the study, the diverse payer mix may have indirectly affected the outcome measure as different levels of coverage, copay-structures of different insurance systems may unduly have affected adherence to therapy.

Retrospective database research studies need to explicitly state the contents of data for the reader to be able to assess the absence of key variables that can affect the outcome measure. ${ }^{3}$ In this case, for instance, since the underlying premise was that the relatively better side-effect profile of the angiotensin receptor blockers (ARBs) compared with the angiotensin-converting enzyme inhibitors and the calcium channel blockers may result in better adherence and persistence with ARBs, it would have been important to know the dosage provided for each of the therapy groups. Generally, most pharmacy databases include drug name, dose, quantity dispensed at each fill, and the dates of the fills.

Two different measures of adherence were used, one that has the duration of therapy as the denominator (called compliance) and the other that has 365 days as the denominator (called medication possession ratio [MPR]). Although there was only a $2 \%$ difference in the compliance measure, there was almost an $8 \%$ to $10 \%$ difference in the MPR measure. This difference has to be viewed with respect to the manner in which the MPR was measured. The authors state that "a patient may demonstrate high compliance but a lower MPR if the patient discontinues therapy at some point within the 12 months postinitiation of therapy."1,.425 Measurement of MPR, however, has not been explicitly defined in terms of whether discontinuers were included in the MPR calculation. If one looks at the compliance measure, MPR measure, and the proportion of discontinuers in each group, then it seems that discontinuers were included, resulting in a higher MPR for the ARB group because of the lower number of discontinuers.
For the persistence measure, these results emphasize the low discontinuation rate associated with being on the ARBs. In terms of adherence (compliance measure), however, there is only a $2 \%$ difference. Elaboration of the clinical significance of this difference is needed. Does this $2 \%$ difference translate into lower costs and improved blood-pressure control? Given that other studies ${ }^{4,5}$ have already shown better adherence profiles for the ARBs, it begs the question as to whether there was really a need for this study. Perhaps the focus should now be on interpreting the meaning of the improved adherence.

Anna O. D'Souza, MS

PhD Student/Aventis Fellow

Lesley Ann Miller, PhD

Assistant Professor

Department of Pharmaceutical Systems and Policy

P.O. Box 9510

West Virginia University

Morgantown, WV 26506

E-mail:adsouza@hsc.wvu.edu

\section{DISCLOSURES}

The authors state that they have no conflict of interest or any potential bias regarding this letter.

\section{REFERENCES}

1. Steiner JF, Prochazka AV. The assessment of refill compliance using pharmacy records: methods, validity and applications. J Clin Epidemiol. 1997;50(1): 105-16.

2. Wogen J, Kreilick, CA, Livornese, RC, Yokoyama K, Frech F. Patient adherence with amlodipine, lisinopril, or valsartan therapy in a usual-care setting; J Manag Care Pharm. 2003;9(5):424-29.

3. Motheral B, Brooks J, Clark MA, et al. A checklist for retrospective database studies. Report of the ISPOR task force on retrospective databases. Value Health. 2003;6(2):90-97

4. Marentette MA, Gerth WC, Billings DK, Zarnke KB. Anti-hypertensive persistence and drug class. Can J Cardiol. 2002;18:649-56.

5. Conlin PR, Gerth WC, Fox J, Roehm JB, Boccuzzi SJ. Four-year persistence patterns among patients initiating therapy with the angiotensin II receptor antagonist losartan versus other anti-hypertensive drug classes. Clin Ther. 2001;23:1999-2010.

\section{The Authors Respond}

Ms. D'Souza and Dr. Miller raise some interesting points regarding our study, "Patient Adherence With Amlodipine, Lisinopril, or Valsartan Therapy in a Usual-Care Setting." We would like to take the opportunity to respond to their comments in this letter.

Assessment of patient copay was not included in the original scope of the study so we cannot rule out the possibility that this had an impact on adherence results. However, one of the benefits of the large size of the pharmacy benefits manager data- 
base used is that many different employer, retirement, and government plans were included, with many and vastly different drug benefit designs most likely represented in the patient mix. This diversity in benefit designs and associated drug copays is likely a strength of our study. In addition, as we pointed out in our response to Dr. Curtiss' editorial (page 90), there is no consensus regarding the impact of patient copay on therapy persistence and adherence. Some studies have found an impact of patient copay on therapy discontinuation, ${ }^{2}$ while others have failed to find an association., ${ }^{3,4}$

We did not examine dosage for each of the agents included in the study, and it is theoretically possible that this may have had some impact on study results. Since dosages are not equivalent across study agents, this would have added considerable complexity to the study analyses and made interpretation of results difficult. Examination of the prescribing information reveals that for lisinopril and valsartan, no dose-related responses were seen for adverse experiences or drug discontinuation in clinical trials. ${ }^{5}$ For amlodipine, some side effects (edema, dizziness, flushing, palpitation) were found to be dose related. ${ }^{5}$ We cannot therefore exclude the possibility that daily drug dosage may have impacted adherence or persistence, at least for amlodipine patients.

One of the strengths of our study was that we did, in fact, use 2 distinct measures of adherence to examine the issue from different perspectives. We referred to "compliance" as the proportion of time that a patient had drug supply "on hand" to the patient-specific duration of therapy, as measured by the last prescription fill date minus the first prescription fill date. This measure, by definition, can only be calculated for those patients with at least 2 fills of the index prescription. Medication possession ratio (MPR) is calculated for all patients using the available follow-up (365 days for patients in the current study). Thus, patient persistence with therapy is reflected in the MPR calculation but not in the compliance calculation. The results of our study indicated significantly greater patient persistence with valsartan as compared with amlodipine or lisinopril, as reflected primarily in persistence rates and improved MPR. This is evident in a comparison of mean length of therapy, as valsartan patients remained on therapy about a month longer (mean $=$ 270.1 days of therapy with valsartan as compared with 241.6 for amlodipine and 234.6 with lisinopril) than patients receiving amlodipine or lisinopril. We believe that this has important implications and agree that further research is warranted to fully explore the potential impact on blood pressure control and clinical outcomes.

D'Souza and Miller question the relevance of our study, citing that other studies have shown improved adherence profiles for angiotensin-receptor blockers (ARBs). ${ }^{6,7}$ However, this is not an entirely accurate interpretation of these studies as they, in fact, investigated medication persistence, not adherence, and neither study was specific to valsartan. No other studies, to date, have investigated valsartan adherence and persistence. Furthermore, as valsartan is currently the most frequently prescribed ARB in the United States, we feel that this is a relevant and timely contribution to the literature.

\section{Jenifer Wogen, MS Manager, Health Economics and Outcomes Research Novartis Pharmaceutical Corporation One Health Plaza East Hanover NJ 07936}

formerly Director, Health Outcomes Research, The Institute for Effectiveness Research, LLC, Bridgewater, NJ)

E-mail: jenifer.wogen@pharma.novartis.com

Feride Frech, $R P h, M P H$

Associate Director, Health Economics and Outcomes Research Novartis Pharmaceutical Corporation, East Hanover, NJ

\section{DISCLOSURES}

Funding for this research was provided by Novartis Pharmaceutical Corporation, via a grant to the Institute for Effectiveness Research, a subsidiary of Medco Health Solutions, Inc., and was obtained by author Jenifer Wogen. Wogen was employed by the Institute for Effectiveness Research at the time the article was written; she is currently employed by Novartis as is Feride Frech. Authors Charles A. Kreilick and Richard C. Livornese are currently employed by the Institute for Effectiveness Research, and author Krista Yokoyama is employed by WellPoint Pharmacy Management. At the time this study was conducted, Yokoyama was a University of Texas/Novartis research fellow.

\section{REFERENCES}

1. Wogen J, Kreilick CA, Livornese RC, Yokoyama K, Frech F. Patient adherence with amlodipine, lisinopril, or valsartan therapy in a usual-care setting. J Manag Care Pharm. 2003;9(5):424-29.

2. Huskamp HA, Deverka PA, Epstein AM, et al. The effect of incentive-based formularies on prescription-drug utilization and spending. N Engl J Med. 2003;349:2224-32.

3. Motheral B, Fairman KA. Effect of a three-tier prescription copay on pharmaceutical and other medical utilization. Med Care. 2001;39:1293-1304.

4. Motheral BR, Henderson R. The effect of a copay increase on pharmaceutical utilization, expenditures, and treatment discontinuation. Am J Manag Care. 1999;5:1383-94

5. Physicians Desk Reference. 56th ed. Montvale, NJ: Medical Economics Company, Inc.; 2002.

6. Marentette MA, Gerth WC, Billings DK, Zarnke KB. Anti-hypertensive persistence and drug class. Can J Cardiol. 2002;18:649-56.

7. Conlin PR, Gerth WC, Fox J, Roehm JB, Boccuzzi SJ. Four-year persistence patterns among patients initiating therapy with the angiotensin II receptor antagonist losartan versus other anti-hypertensive drug classes. Clin Ther. 2001;23:1999-2010.

8. IMS America data, October 2003. 


\section{Patient Adherence With Hypertension Medication}

To the Editor:

We are writing to discuss the paper entitled "Patient Adherence With Amlodipine, Lisinopril, or Valsartan Therapy in a Usual-Care Setting"1 and the subsequent editorial, "Hypertension, Prescription Drug Copayments, and Drug Therapy Adherence,"' both published in the September/October 2003 issue of the Journal of Managed Care Pharmacy. Our retrospective database study found increased patient adherence and persistence with valsartan, an angiotensin-receptor blocker (ARB), as compared with the most frequently prescribed agents from the calcium channel blocker (CCB - amlodipine) and angiotensin-converting enzyme inhibitor (ACEI-lisinopril) classes, controlling for available patient baseline factors, including age, gender, and baseline comorbid conditions. We welcome the thoughtful evaluation and suggestions in the editorial and would like to clarify some of the issues that were raised.

As Dr. Frederic Curtiss mentioned in his editorial, plan information such as patient copay and drug formulary status was not included in the study results. His editorial discusses the small increase in U.S. sales of atorvastatin and decrease in sales of sertraline and amlodipine in Q1 2003 and analysts' speculation of a relationship to rising drug plan copayments as published in the Wall Street Journal ${ }^{3}$ and suggests that patient copayment amounts and drug formulary status, which were not assessed in the study, may have impacted the study results. Recently, many insurers have adopted incentive-based ("tiered") formularies for prescription medication coverage. For example, a patient with a 3-tier formulary typically has the lowest copay for generic drugs (the first tier), a higher copay for preferred brand-name drugs (second tier), and the highest copay for third-tier brand-name drugs.

Relatively few peer-reviewed studies have been published examining the relationship between patient copay for pharmaceutical agents and associated patterns of therapy compliance and persistence. Results of a recent study using pharmacy claims data suggested increased ACEI, proton-pump inhibitor, and statin discontinuation rates subsequent to the implementation of a 3-tier copay formulary in a plan that had previously had a 1-tier structure as compared with a comparison plan with a stable 2-tier structure. ${ }^{4}$ However, the same study found no increase on discontinuation rates for the same classes of drugs in a plan that implemented a less-drastic change in formulary structure (2-tier to 3-tier). Another study utilized an integrated medical and pharmacy claims database to examine drug continuation rates for members for which a 3-tiered copay system was implemented as compared with a control group that remained on a 2-tiered benefit and found significantly lower therapy continuation rates for only 1 therapeutic class out of 4 studied. ${ }^{5}$ A different study found no increase in chronic branded medication discontinuation for patients with copay increases from $\$ 10$ to 15 as compared with patients whose copay amount remained fixed at $\$ 10 .{ }^{6}$ Thus, there is a lack of clear evidence in the peer-reviewed literature regarding the hypothesis that increased patient copay results in greater medication dis- continuation rates or patient noncompliance.

The editorial inaccurately stated that one of the study medications, lisinopril, became available as a generic at the end of the study; in fact, the study analyzed data through July 31, 2000, and generic lisinopril did not become available in the United States until July 2002. While the introduction of generic lisinopril did not impact the results of this study, the introduction of generic ACEIs is an undeniably important factor for current studies of the hypertension market.

Curtiss also pointed out an important observation, namely that channel of delivery was not included in the published study. Members of most prescription plans are often limited to a 30-day supply of medication at a retail pharmacy but may often receive up to a 90-day supply if they receive the medication via home delivery. Thirty-four percent of patients included in the study filled one or more study medications via mail service (data not presented in our article). As would be expected, these patients were more adherent with the study agent than patients who received all study prescriptions via retail service only (overall medication possession ratio [MPR], mean of $59.7 \%$ for retail-only patients versus $85.3 \%$ for patients with one or more mail prescriptions). However, valsartan patients consistently demonstrated a higher mean MPR than patients prescribed amlodipine or lisinopril. Valsartan patients with one or more mail fills had a mean MPR of $89.0 \%$ compared with $84.2 \%$ for amlodipine and $83.8 \%$ for lisinopril; valsartan patients who received all fills via a retail pharmacy had a mean MPR of 66.1\% compared with $57.9 \%$ for amlodipine and $58.6 \%$ for lisinopril. Thus, we agree that channel of prescription delivery can play an important role in patient adherence with therapy, though it was not directly addressed in the scope of our original study.

Information about blood-pressure control was not available for this study due to the nature of the administrative claims database employed, and this is an important study limitation. While research has shown that persistence with antihypertensive therapy is associated with lower rates of adverse long-term sequelae of hypertension ${ }^{7}$ and decreased health care resource consumption, ${ }^{8}$ there is little that is documented about the implied relationship between therapy compliance and persistence and their impact on blood pressure control in a usual-care setting. Additional research is needed to explore the relationship of the current study findings and others demonstrating superior compliance with ARBs to blood pressure control in a nonclinical trial setting.

In addition, the authors agree with Curtiss that the information provided by this study must be applied in the context of recent clinical studies. ALLHAT ${ }^{9}$ followed 33,357 patients for a mean of 4.9 years and found that patients treated with amlodipine or lisinopril had the same incidence of combined fatal coronary heart disease or nonfatal myocardial infaction as a thiazide diuretic (chlorthalidone), but patients treated with the diuretic had lower rates of heart failure at 6 years than amlodipine patients, while lisinopril patients had higher 6-year rates of combined cardiovascular disease, stroke, and heart failure. Patients treated with chlorthalidone had a higher incidence of 
diabetes at 2 and 4 years of follow-up as compared with amlodipine and lisinopril patients. The Second Annual Australian Blood Pressure (ANBP2) study ${ }^{10}$ found results that are somewhat contradictory; in this study, which followed 6,083 patients for a mean of 4.1 years, the investigators reported reductions in the rates of total cardiovascular events, first cardiovascular event, and all-cause mortality for patients treated with ACEI as compared with diuretics. What are the differences between the 2 trials? ANBP2 used enalapril as the ACEI and hydrochlorothiazide as the diuretic, while ALLHAT used lisinopril and chlorthalidone. There is no information regarding a comparison of clinical efficacy of enalapril versus lisinopril or chlorthalidone versus hydrochlorothiazide, and there is not likely to ever be any. ${ }^{11}$ Patients in ANBP2 were older (mean = 71.9 years versus 67 years for ALLHAT patients), had higher mean diastolic and systolic blood pressure readings at study baseline $(168 \pm 13 / 91 \pm 8$ versus $146 \pm 16 / 84 \pm 10$ for ALLHAT), were more likely to be white than black or Hispanic (95\% versus $46 \%$ for ALLHAT), and were less likely to have diabetes ( $7 \%$ versus $36 \%$ for ALLHAT). Thus, these critical differences in study populations and medications employed make comparing the results difficult and a definitive statement regarding the superiority of an ACEI versus a thiazide diuretic problematic.

Nevertheless, patient behavior during participation in a randomized trial differs from patient behavior in a real-world setting, where patients may not be as motivated to take prescribed medication exactly as instructed by their health care providers. It is in this context that studies such as the current one become important. While the authors agree that a difference in mean compliance (measured as the sum of total days' supply divided by the length of therapy) of $88.5 \%$ for valsartan as compared with $86.7 \%$ for amlodipine or $86.3 \%$ for lisinopril may be statistically significant only due to the very large sample size, the paper reported a greater length of therapy with valsartan compared with the other study agents that the authors believe is clinically very meaningful. This is reflected in both increased persistence rates, as $63 \%$ of valsartan patients remained on therapy at 12 months compared with 53\% and 50\% for amlodipine and lisinopril patients, respectively, and higher medication possession ratio for valsartan (mean $=75 \%$ ) as compared with amlodipine (67\%) or lisinopril (65\%) patients. This is most evident in the study finding that patients stayed on therapy an average of a month longer with valsartan as compared with the other agents (mean = 270.1 days of therapy with valsartan as compared with 241.6 for amlodipine and 234.6 with lisinopril), which has the potential to positively impact patient outcomes.

Jenifer Wogen, MS

Manager, Health Economics and Outcomes Research Novartis Pharmaceutical Corporation One Health Plaza East Hanover, NJ 07936

(formerly Director, Health Outcomes Research, The Institute for
Effectiveness Research, LLC, Bridgewater NJ)

E-mail: jenifer.wogen@pharma.novartis.com

Feride Frech, RPh, MPH

Associate Director, Health Economics and Outcomes Research Novartis Pharmaceutical Corporation, East Hanover, NJ

\section{DISCLOSURES}

See page 89 for Wogen's and Frech's disclosure information.

\section{REFERENCES}

1. Wogen J, Kreilick CA, Livornese RC, Yokoyama K, Frech F. Patient adherence with amlodipine, lisinopril, or valsartan therapy in a usual-care setting. J Manag Care Pharm. 2003;9(5):424-29.

2. Curtiss FR. Hypertension, prescription drug copayments, and drug therapy adherence. J Manag Care Pharm. 2003;9(5):454-56.

3. Hensley S, Burton TM. Big drug makers report increase in sales. Wall Street Journal. April 23, 2003:B2.

4. Huskamp HA, Deverka PA, Epstein AM, et al. The effect of incentive-based formularies on prescription-drug utilization and spending. N Engl J Med. 2003;349:2224-32.

5. Motheral B, Fairman KA. Effect of a three-tier prescription copay on pharmaceutical and other medical utilization. Med Care. 2001;39:1293-1304.

6. Motheral BR, Henderson R. The effect of a copay increase on pharmaceutical utilization, expenditures, and treatment discontinuation. Am J Manag Care. 1999;5:1383-94.

7. Cramer JA. Consequences of intermittent treatment for hypertension: the case for medication compliance and persistence. Am J Manag Care. 1998:4: 1563-68

8. McCombs JS, Nichol MB, Newman CM, Sclar DA. The costs of interrupting antihypertensive therapy in a Medicaid population. Med Care. 1994:32:214-26.

9. The ALLHAT Officers and Coordinators for the ALLHAT Collaborative Research Group. Major outcomes in high-risk hypertensive patients randomized to angiotensin-converting enzyme inhibitor or calcium channel blocker vs. diuretic: the Antihypertensive and Lipid-Lowering Treatment to Prevent Heart Attack Trial (ALLHAT). JAMA. 2002;288:2981-97.

10. Wing LMH, Reid CM, Ryan P, et al. A comparison of outcomes with angiotensin-converting-enzyme inhibitors and diuretics for hypertension in the elderly. N Engl J Med. 2003;348:583-92.

11. Frohlich ED. Treating hypertension-what are we to believe? N Engl J Med. 2003;348:639-41.

\section{Letters to the Editor}

$J M C P$ welcomes letters that serve to clarify subjects published in previous issues of the Journal or regarding subject matter of interest to managed care pharmacists. Letters in JMCP are not peer reviewed but are subjected to editorial review. When a submitted letter refers to an article published in a previous issue of the Journal, the letter is sent to the authors of the subject article to allow their response to be published with the letter.

Each letter should be signed by no more than 3 authors. Submissions must include your title, affiliation, complete mailing address, telephone number, and e-mail address. Potential bias or conflicts of interest must be disclosed.

Letters should be submitted in electronic format, preferably using Microsoft Word, and may be sent by e-mail to Fred Curtiss, editor-inchief, at fcurtiss@amcp.org or to Tamara Faggen, managing editor, at tfaggen@amcp.org. 